\title{
Pigs in a Blanket: Evaluation of a Novel Device to Support Premature Neonatal Thermal Regulation
}

\author{
Grant Tomlin ${ }^{1}$, Nicholas David - Paul Bluhm², Lagora Carrell ${ }^{2}$, Melissa Bible ${ }^{2}$, Jacqueline \\ Linnes $^{2}$ \\ ${ }^{1}$ Indiana University School of Medicine; ${ }^{2}$ Purdue University, Weldon School of Biomedical \\ Engineering
}

Background: 1.47 million preterm births worldwide tragically end in neonatal death (death within 28 days after birth). Contributing to this is the inability for preterm neonates to thermoregulate. In low-resource areas, access to incubators may be non-existent. Kangaroo Mother Care/Skin-to-Skin care (KMC/STS) is one method used in such situations and involves the caregiver strapping the infant to their chest in a kangaroo-like pouch. This method is effective but does not provide warming when the caregiver needs rest, nor does it allow the infants heart and respiratory rate to be monitored reliably. We created a device that aims to solve these issues in a safe, cost effective, and efficient way.

Methods: Pilot studies were performed with 3 pigs to develop parameters that will validate the devices efficacy in a pre-clinical trial. Newborn pigs from 0-5 days old were used as the model for these initial trials. Test configurations were designed to demonstrate 1) the pigs could not regulate their core temperature without the device and 2) the heating elements of the device provided better support than a simple blanket. The pig's core temperature was used as the standard measurement across all trials.

Results: When exposed to an ambient temperature of $20^{\circ} \mathrm{C}$, the pigs were not able to regulate their core temperature, which dropped $0.5^{\circ} \mathrm{C}$ per hour. After reaching hypothermic temperature (below $36.5^{\circ} \mathrm{C}$ ), pigs placed in the warming device returned to a healthy core temperature $\left(38.6^{\circ} \mathrm{C}\right)$ over the course of one hour. Pigs in the device with heating off only recovered partially. No pig had any abrasions or burns.

Conclusion: These studies prove the devices feasibility and have established the parameters needed to design a larger trial. If the device continues to show similar efficacy in the future tests, it could be used to help lower premature infant mortality in low-resource settings. 